\title{
Applications of SEM/FIB Technology in Life Science
}

\author{
A. Schertel, D. Kraft, M. Rauscher \\ Carl Zeiss NTS GmbH, Carl-Zeiss-Str.56, D-73447 Oberkochen, Germany
}

In combined SEM/FIB systems material removal with FIB milling is employed to open up the third dimension for SEM imaging. Applications include precise site-specific cross-sectioning, TEM sample preparation and completely automated slicing for 3D reconstruction of regions of interest on a nanometer level [1].

In materials science and semiconductor industry FIB/SEM technology is extensively used and very well established for imaging and analysis of structure and composition of materials down to the nanometer scale. However, recently there has been an increasing interest in also benefiting from the capabilities of SEM/FIB technology in life science applications. Focus topics of interest are cell adhesion to substrates, bio-compatibility (implants), 3D reconstruction of site-specific regions of interests visualizing the ultrastructure (e.g., 3D brain mapping $[2,3]$ ).

For electron microscopy, the conventional preparation of biological samples is to cut very thin section of tissues embedded in epoxy by a glass or diamond knife using a microtome. With this preparation technique only soft materials can be cut and the interface between silicon structures or hard materials and the soft biological tissue is not accessible. Compared to the thickness of TEM sections $(\mathrm{d} \leq 100 \mathrm{~nm})$ typical biological are large. A single cell has a size of 10-20 $\mu \mathrm{m}$. For 3D reconstruction of the complete cell about 100 - 200 TEM sections have to be prepared and analyzed. Reliable serial slicing requires a thin section thickness of about $40 \mathrm{~nm}$ or thicker limiting the $\mathrm{z}$ resolution for $3 \mathrm{D}$ reconstruction. Below about $40 \mathrm{~nm}$ thickness the risk of introducing cutting artefacts escalates.

In contrast, using SEM/FIB technology site specific regions of interest near the surface of biological samples embedded in epoxy can be directly cross-sectioned and imaged for 3D reconstruction in a completely automated process. By low $\mathrm{kV}$ surface imaging, detecting the low loss backscattered electrons of the stained tissue embedded in epoxy, TEM like contrast after inversion of greyscale and comparable image information is achieved. The z-resolution is given by the FIB slice thickness which can be much less than $40 \mathrm{~nm}$.

In this paper we will present application examples showing the benefits of the SEM/FIB approach for analysis and 3D reconstruction of biological samples (e.g. fig. 1 and 2).

References

1. H. Hoffmeister, A. Schertel, A. Thesen, P. Gnauck, Proc. M\&M 2006, Microsc. Microanal. 12, Supp. S02, 1244CD (2006).

2. J. DeFelipe, Front. Neurosci. 2, 1: 10-12 (2008)

3. G. Knott, H. Marchman, D. Wall, B. Lich, J. Neurosci. 28, 2959-2964 (2008) 


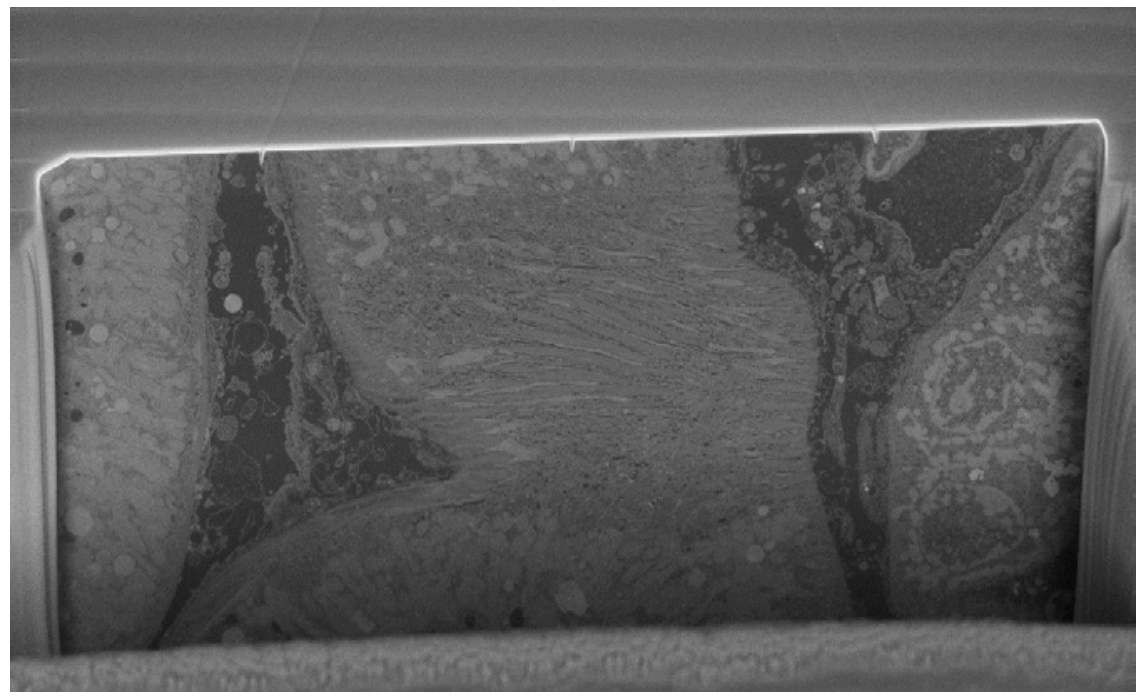

Figure 1: Low kV in-lens back-scattered electron imaging: FIB cross-section ( $60 \mu \mathrm{m}$ wide and 30 $\mu \mathrm{m}$ deep) of kidney tissue embedded in epoxy (sample courtesy of Mario Negri Institute, Bergamo, Italy).

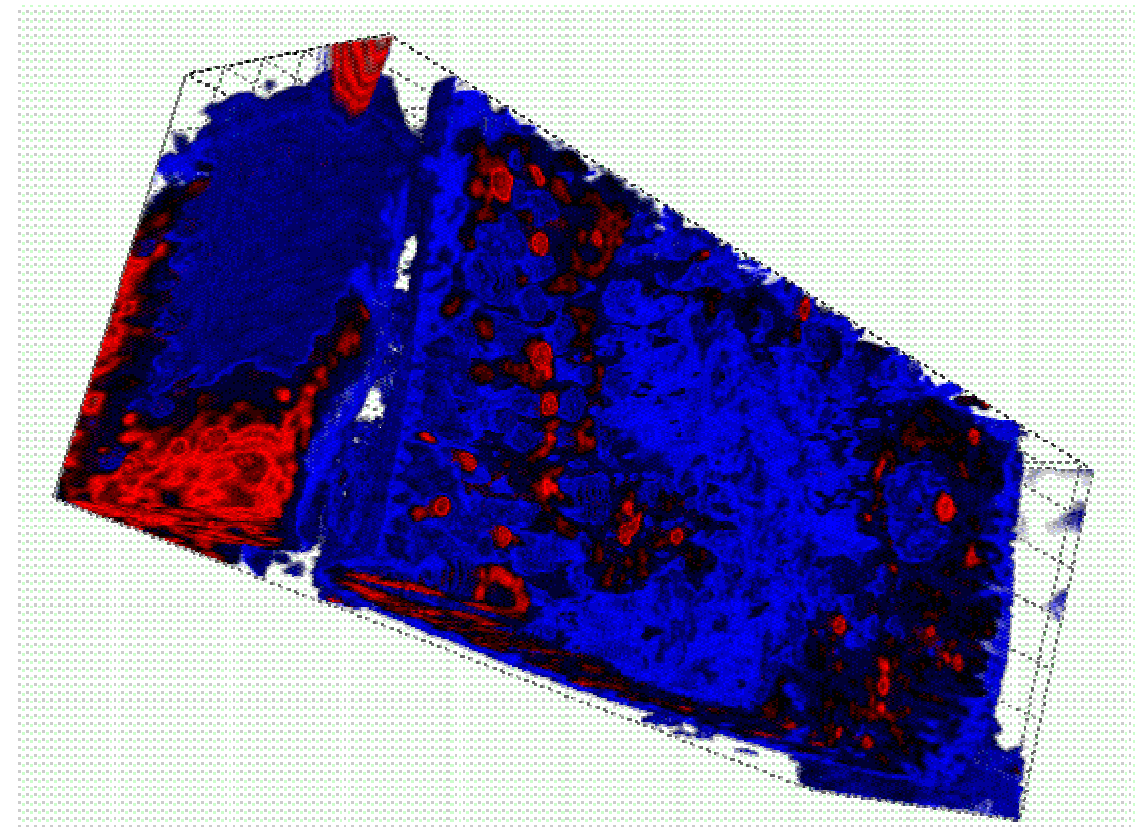

Figure 2: 3D reconstruction of kidney tissue based on low $\mathrm{kV}$ low loss BSE images as shown in fig. 1. 